\section{Unfit for modern life}

\author{
Mismatch: Why Our World No Longer Fits \\ Our Bodies \\ by Peter Gluckman \& Mark Hanson \\ Oxford University Press: 2006. 304 pp. \\ E16.95, \$29.95
}

\section{Michael Sargent}

Will people born in the developed world in the 1990s have shorter lives than those born 60 years ago? If the fears of the authors of the thought-provoking book Mismatch are realized, the upward trend in life expectancy over the past century is set to reverse unless the lifestyle of young people dramatically improves. The authors, Peter Gluckman and Mark Hanson, are not alone in their anxiety: some US experts on longevity believe that the steady rise of childhood obesity in their country is likely to reduce life expectancy by 2 to 5 years by the middle of the century, with enormous increases in morbidity from type 2 diabetes, heart disease and other vascular disorders.

Curiously, while the horror of this impending public-health disaster lies in the future, the authors overlook the tragedy already afflicting the Pima Indians of Arizona and the inhabitants of the Pacific island of Nauru. Just a few decades ago, these populations were lean and fit and knew nothing of diabetes; now the majority of young adults become obese, develop diabetes and die prematurely of heart disease. A genetic predisposition to diabetes may have underpinned the crisis, but the immediate cause was undoubtedly an abrupt decline in strenuous physical activity along with the displacement of the traditional cuisine by energydense food.

Recognizing the emergence of a global epidemic of a similar kind, Gluckman and Hanson condemn the mismatch between the intrinsic physiological capacities programmed in utero and our twenty-first-century lifestyles, which encourage the consumption of excess calories without the physical demands of former times. With Old Testament severity the authors call for "a return to a different way of life" and condemn the modern habitat, to which they feel we are poorly adapted - a counterintuitive notion at a time when life expectancy is higher than ever.

The heart of the book is predicated on David Barker's idea that nutritional and other kinds of stress impinging on pregnant women may register in utero and affect the postnatal development of progeny. Extensive investigations of laboratory mammals and human epidemiology portray the response to nutritional stress as a kind of faustian bargain. The cost of protecting the brain and reproductive behaviour is underdeveloped viscera that may compromise health and survival in later life. There is a greater burden if the nutritional experiences of the offspring are richer than their mother's during pregnancy, with greater risks of premature onset of the chronic diseases of middle age: coronary heart disease, type 2 diabetes, hypertension and osteoporosis. The embryo is also sensitive to excessive nutrition from high maternal blood sugar and psychological stress, resulting in epigenetic modifications of genomic function that are expressed after birth in surprising ways in humans and animals.

The average stature and physique of humans from different parts of the world reflect the local nutritional regime by processes that are established in utero and transmitted epigenetically to succeeding generations. It seems that obesity and its consequences are likely to emerge when this regime changes. In the developing world, those released from the grip of historic poverty are most affected, whereas in the developed world, for reasons that are less well understood, the victims are the least educated and the least affluent. The authors believe that research should focus on improving maternal nutrition to ensure that babies are better adapted to changing nutritional landscapes.

Gluckman and Hanson also apply their 'mismatch paradigm' to a diverse group of situations in which cultural developments that challenge the logic of darwinian evolution generate new problems. These include the emergence of a long post-reproductive life, and the tricky interval between sexual maturity and the time when young adults are considered mature enough, for example, to hire a car. Other issues include attitudes to breastfeeding, the effect of breeding patterns on cancers of the reproductive organs, living with micronutrient deficiencies, and the onset of myopia provoked by excessive reading at an early age. Other authors mining the same vein have unearthed more issues of human caprice and mismatches of the kind the authors find so disturbing.

The authors sometimes skim over human biology, preferring to explore the natural history of other species. I was amused by their conflation of Psalm 90 with the Gettysburg address, and horrified by persistent references to the 'design' of organisms - a usage notably obstructive to an understanding of the evolutionary process, the disclaimer notwithstanding. Overall, however, this book conveys admirably, for a non-specialist reader, the implications of an important idea.

Michael Sargent is a developmental biologist at the National Institute for Medical Research, Mill Hill, London NW7 1AA, UK. He is the author of Biomedicine and the Human Condition: Challenges, Risks, and Rewards.

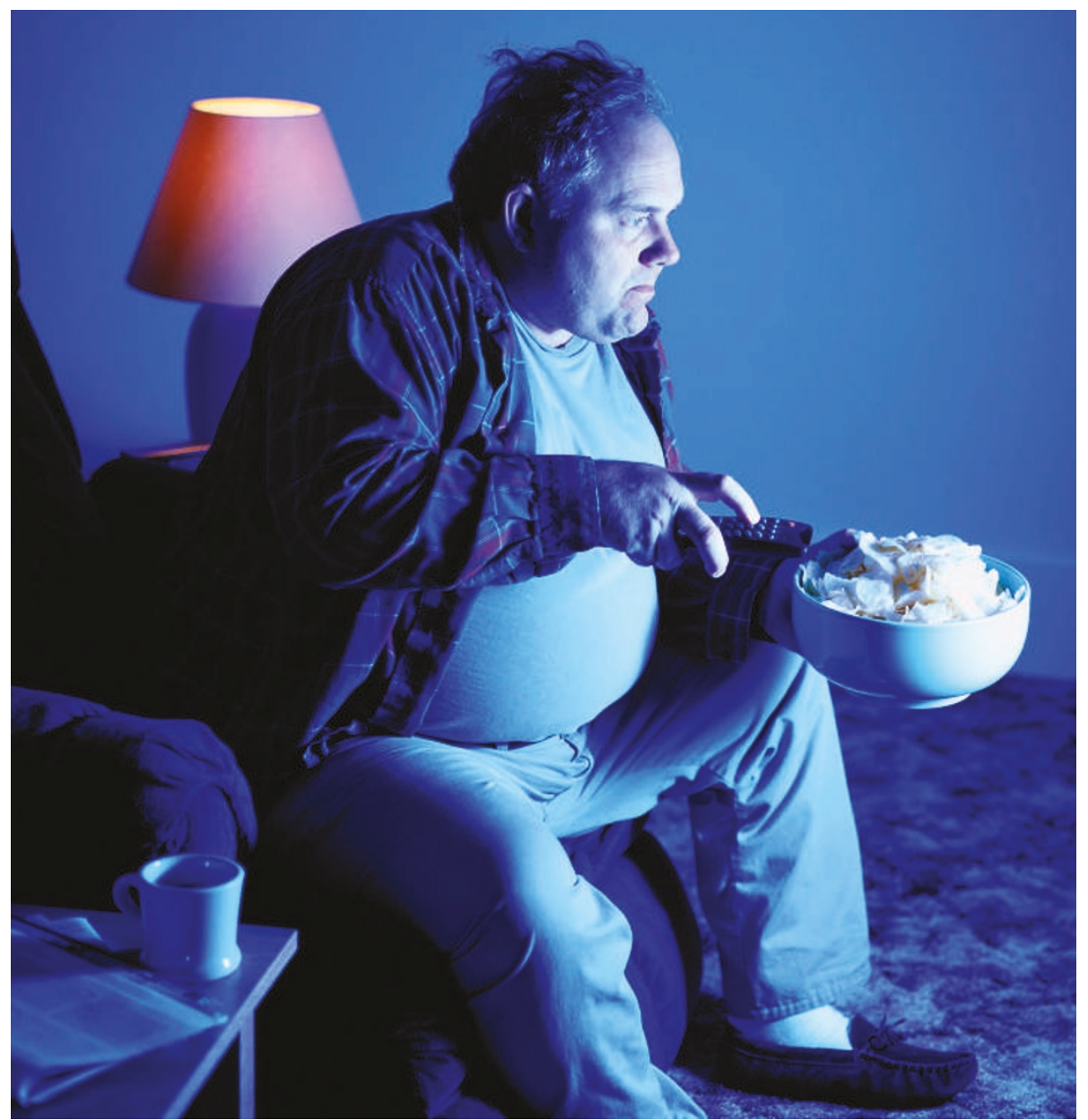

The fat of the land: obesity stems from our failure to adapt to an increasingly high-calorie diet. 